\title{
Hitting the right button: MAVS-mediated defense against HAV infection
}

Cell Research (2017) 27:7-8. doi:10.1038/cr.2016.139; published online 25 November 2016

Studying the immune response against infection with hepatitis viruses is hampered by the lack of suitable preclinical model systems. A recent publication in Science identifies the cytosolic adapter molecule MAVS as being responsible for species restriction of infection with hepatitis A virus as well as linking cytosolic immune sensing in infected hepatocytes with innate effector functions and protective adaptive immunity.

Infection with hepatitis viruses, such as hepatitis A virus (HAV), hepatitis B virus (HBV) or hepatitis $\mathrm{C}$ virus (HCV) is restricted to humans and chimpanzees. Studying the molecular and cellular mechanisms determining viral clearance or persistence within the unique immunological microenvironment of the liver is hampered by the lack of suitable preclinical model systems [1]. Different hurdles exist for hepatitis viruses to infect hepatocytes in genetically defined preclinical models such as mice [2]. HBV does not infect mice because its receptor is not expressed on hepatocytes but HBV replication can occur within hepatocytes albeit not from the natural viral template, the HBV covalently-closed-circular DNA (cccDNA) [2]. Thus, preclinical models of HBV infection rely on transgenic mice or viral gene transfer of HBV genomes into hepatocytes. $\mathrm{HCV}$ also fails to infect hepatocytes in mice but transgenic expression of its receptors renders mice susceptible to HCV infection [3]. In contrast, HAV infects murine cells in vitro but fails to establish liver infection in mice. HAV replication is suppressed by type-I-interferons. Yet, HAV encodes a protease that degrades the intracellular signal adapter proteins MAVS and TRIF that mediate induction of type-I-interferon, thereby blunting interferon responses in infected hepatocytes.

Hirai-Yuki et al. [4] now report that HAV can infect mice that lack type-IInterferon signaling, i.e., IFN-receptor (IFNAR) knockout mice. Such infection is similar to HAV infection in humans and is characterized by fecal HAV shedding, prolonged infection over three months with low-grade viremia and liver damage. Thus, evading typeI-Interferon production defines the host range of HAV. Detection of viral constituents such as nucleic acids through the cytosolic immune sensors RIG-I or MDA5 is responsible for induction of type-I-interferons by signaling through the adapter molecule MAVS (see Figure 1). The authors demonstrate that HAV $3 \mathrm{ABC}$, a protease degrading human MAVS, does not target murine MAVS and therefore fails to counteract typeI-interferon induction in HAV-infected hepatocytes.

Consequently, in MAVS-knockout mice, HAV also establishes liver infection, but two important differences are observed compared to IFNAR-knockout mice. HAV replication in the liver was 10 -fold higher and was not accompanied by liver damage any more. HAV-induced liver damage was not mediated by immune effector cells such as CD8 T cells or NK cells indicating that activation of MAVS itself initiates the apoptosis of infected hepatocytes.
This demonstrates that MAVS serves two distinct functions in HAV-infected hepatocytes: first, it triggers type-IInterferon production that is responsible for controlling HAV replication in infected hepatocytes and defines the species barrier; and second, it initiates death of HAV-infected hepatocytes. In search of the mechanism mediating MAVS-induced death, the authors found that HAV-induced death of infected hepatocytes depends on the transcription factors IRF3 and IRF7. Since HAVinduced liver damage is independent of IFNAR signaling, IRF3/7 may trigger death directly through its transcriptional activity on pro-apoptotic molecules like the BH3-only protein PMAIP1 [5] or in a transcription-independent fashion by interacting with mitochondrial Bax [6]. Hirai-Yuki et al. provide compelling evidence in their study for the key role of MAVS in the control of hepatocyte infection with an RNA virus like HAV. Infection by DNA viruses, on the other hand, is detected by the cGAS-STING pathway [7], which also elicits IRF3/7 phosphorylation and type-I-Interferon production (see Figure 1). While there is no evidence for DNA viruses causing STING-mediated damage in infected hepatocytes, alcohol-induced and STING-mediated IRF3 activation can cause hepatocyte apoptosis in the context of ER stress [8]. The mechanisms that determine the outcome of RIG-I/MDA5/MAVS or cGAS/STING activation for induction of cell death remain unclear but may be related to the distinct subcellular localization of MAVS to mitochondria and STING to 


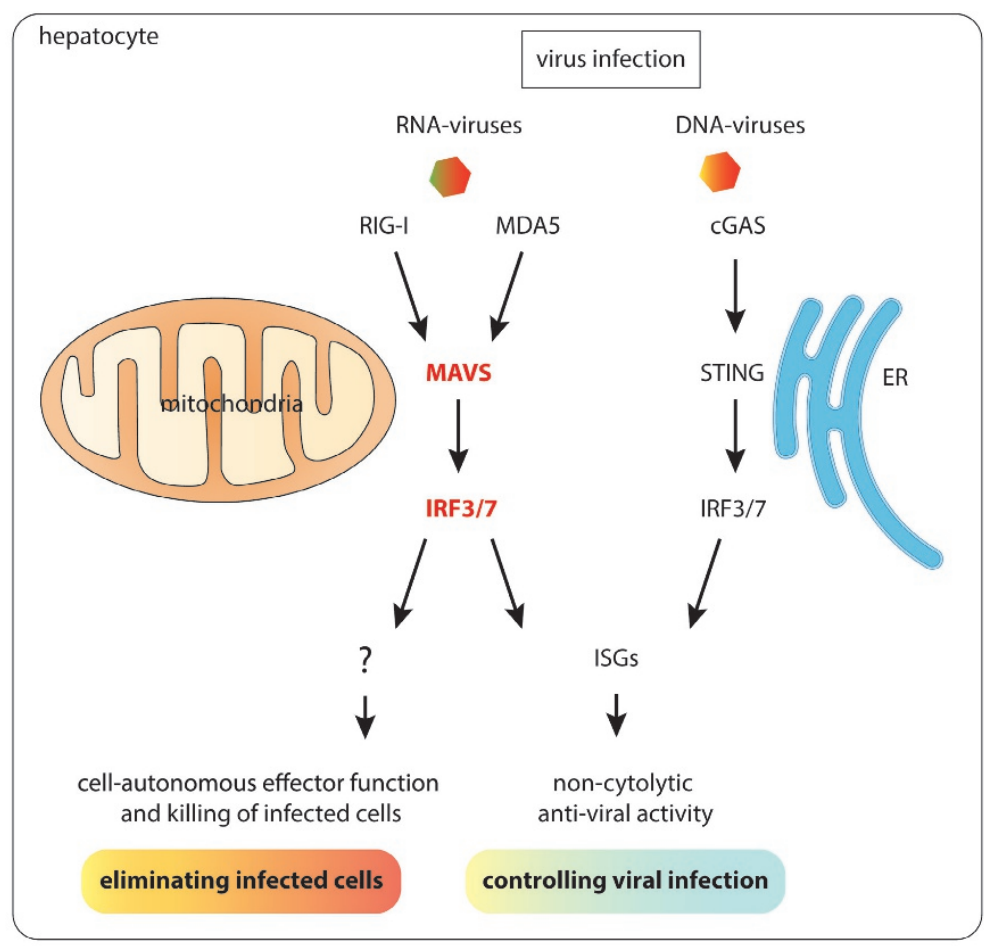

Figure 1 Schematic illustration of cytosolic immune sensors detecting viral infection and signaling through the adapter molecules MAVS or STING to elicit type-l-interferon expression or trigger cell death.

the ER in combination with hepatocyte stress conditions.

The elegant study by Hirai-Yuki et al. reveals that immune control of HAV infection is critically determined by innate immune signaling that also affects adaptive immune responses against HAV. The self-limited course of hepatic HAV infection may be determined by this MAVS-dominated hepatocyteintrinsic innate immune defense. Since necroptotic cell death determines immunogenic activation of antigen-presenting dendritic cells for cross-priming of CD8 $\mathrm{T}$ cells that are necessary for protective immunity against virus infection [9], more details need to be known on the mode of MAVS/IRF3-induced death in HAV-infected hepatocytes and how this translates into protective immunity against HAV. In HBV infection, no viral immune escape in hepatocytes has been reported, and $\mathrm{HBV}$ is rather considered a stealth non-cytopathic virus that evades rather than actively modulates innate immune response [1]. However, a recent study demonstrated that HBV infection upregulates cellular inhibitor of apoptosis proteins (cIAPs) and thereby skews TNF receptor signaling towards survival rather than death in HBVinfected hepatocytes [10]. While viral constituents or viral replication can be revealed by cytosolic immune sensors, it is much more demanding to detect viral persistence forms in hepatocytes, such as HBV cccDNA. Deciphering the crosstalk between hepatitis viruses and innate immune defense systems in hepatocytes will provide important clues to understand how innate immunity is linked to virus-specific adaptive immunity during hepatitis virus infection. Fit-for-purpose preclinical model systems for hepatitis virus infections that reflect the clinical course of disease in humans are needed to advance our knowledge on the relevant mechanisms responsible for immune control of hepatitis virus infection and to test immune intervention strategies to overcome chronic viral hepatitis.

Percy A Knolle ${ }^{1}$

${ }^{1}$ Institute of Molecular Immunology and Experimental Oncology Technische Universität München, Germany

E-mail: percy.knolle@tum.de

\section{References}

1 Protzer U, Maini MK, Knolle PA. Nat Rev Immunol 2012; 12:201-213.

2 Winer BY, Ding Q, Gaska JM, et al. FEBS Lett 2016; 590:1987-1999.

3 Dorner M, Horwitz JA, Donovan BM, et al. Nature 2013; 501:237-241.

4 Hirai-Yuki A, Hensley L, McGivern DR, et al. Science 2016; 353:1541-1545.

5 Besch R, Poeck H, Hohenauer T, et al. $J$ Clin Invest 2009; 119:2399-2411.

6 Chattopadhyay S, Kuzmanovic T, Zhang Y, et al. Immunity 2016; 44:1151-1161.

7 Barber GN. Nat Rev Immunol 2015; 15:760770.

8 Petrasek J, Iracheta-Vellve A, Csak T, et al. Proc Natl Acad Sci USA 2013; 110:1654416549.

9 Yatim N, Jusforgues-Saklani H, Orozco S, et al. Science 2015; 350:328-334.

10 Ebert G, Preston S, Allison C, et al. Proc Natl Acad Sci USA 2015; 112:5797-5802. 